\title{
Reliability and Validity of the Japanese Translated Version of the Swedish Demand-Control-Support Questionnaire
}

\author{
Junji MASE $^{1^{*}}$, Atsuhiko OTA ${ }^{1}$, Ken INOUE ${ }^{1}$, Tadayuki IIDA ${ }^{1}$, \\ Akizumi TSUTSUMI ${ }^{2}$, Hiroshi YATSUYA ${ }^{1}$ and Yuichiro ONO ${ }^{1}$ \\ ${ }^{1}$ Department of Public Health, Fujita Health University School of Medicine, Japan \\ ${ }^{2}$ Department of Public Health, Kitasato University School of Medicine, Japan
}

Received February 15, 2011 and accepted July 4, 2012

Published online in J-STAGE October 8, 2012

\begin{abstract}
Reliability and validity of the Japanese-translated version of the Swedish Demand-Control-Support Questionnaire (J-DCSQ) was examined. The subjects comprised 212 women working in nursery schools. The 17-item J-DCSQ consists of three subscales: psychological demands (PD), decision latitude (DL), and social support (SS). With regard to reliability, one-week test-retest reliability and internal consistency were proved at a sufficient level. Weighted kappa coefficients were near or greater than $\mathbf{0 . 6 0}$ for all items. Intraclass correlation coefficients were greater than $\mathbf{0 . 8 0}$ for all subscales. Cronbach's alphas (mean inter-item correlation) were $0.64(0.26), 0.63(0.22)$, and 0.86 (0.50) for PD, DL, and SS respectively. The scaling and concurrent validity was also established at a satisfactory level. Ceiling or floor effects were not found for any subscales. The J-DCSQ helped us obtain accurate estimates on extreme response distribution. By using the Japanese version of the Job Content Questionnaire for comparison, Spearman's correlation coefficients were estimated to be $0.74,0.60$, and 0.59 for PD, DL, and SS respectively. In contrast, the factorial validity was not shown as originally hypothesized. Factor analysis did not reveal that PD and DL were definitely separated. In addition, both a PD item and a DL item indicated low factor loadings. In conclusion, the present findings exhibited sufficient reliability of the J-DCSQ, while further studies are needed to establish its validity.
\end{abstract}

Key words: Demand-Control-Support Questionnaire, Job Demand-Control-Support model, Psychosocial work environment, Reliability, Validity, Japan

\section{Introduction}

Psychosocial work environment is now regarded as an important occupational health problem because of its impact on personal well-being and its worksite effects such as decreased productivity ${ }^{1}$. The Job Demand-Control-Sup-

*To whom correspondence should be addressed.

E-mail: mase@fujita-hu.ac.jp

(C)2012 National Institute of Occupational Safety and Health port (DCS) model $^{2)}$ is a representative concept in examining the interaction between psychosocial work environment and occupational stress responses. The DCS model focuses on job task profiles and measures the following 3 uncorrelated components: psychological demands (PD), decision latitude (DL), and social support (SS). PD was originally assumed to evaluate quantitative and conflicting demands of work. DL includes decision authority and skill utilization for a task. SS-helpful social interaction in the workplace - is supposed to buffer the effects of a harmful 
psychosocial work environment. In order to assess psychosocial work environment on the basis of the DCS model, the Job Content Questionnaire (JCQ) ${ }^{2)}$ has served as a de facto standard. The questionnaire has been translated into many languages, including Japanese $\mathrm{e}^{3,4)}$.

The Demand-Control-Support Questionnaire (DCSQ) was developed as a shortened and modified version of the JCQ and has been used in a number of epidemiological studies $^{5-13)}$. Although the DCSQ is available in Swedish, English, and other languages, it has not yet been translated into Japanese. Some differences exist between the JCQ and the DCSQ. First, the DCSQ has a smaller number of items than the JCQ (17 vs. 22), possibly enabling easier usability. Second, the DSCQ contains 4 frequency-based response options for evaluating PD and DL (interval scale: 'often', 'sometimes', 'occasionally', and 'never or hardly'), while the JCQ contains response options in the agree-disagree format (nominal scale: 'strongly agree', 'agree', 'disagree', and 'strongly disagree'). This means that, compared to the JCQ, the DCSQ has simpler and more satisfactory response options with unidimensional frequency: zero to positive. Meanwhile, the JCQ also has 4 response options, regardless of the bidimensional direction (agree or disagree; positive or negative) and the intensity (strongly or not) ${ }^{14)}$. Finally, regarding SS, the DCSQ items are oriented towards the atmosphere at the workplace. On the other hand, the JCQ items are more objective and instrumental in nature ${ }^{8)}$. Therefore, the reliability and validity of the JCQ may not be applicable to the DCSQ. A limited number of studies have reported on the internal consistency $^{5,6,8,11,13)}$, test-retest reliability ${ }^{7}$, and factorial validity $^{8,12)}$ of the DCSQ.

The relationship between the DCSQ subscales and person-related outcomes have been reported by the following studies ${ }^{7,9,10)}$. A cross-sectional study investigated the relationships among work-related psychosocial factors, worries about work conditions, and musculoskeletal disorders ${ }^{7)}$. Increased psychological demands and decreased social support were significantly associated with neck-shoulder complaints and activity limitation ${ }^{9}$. Another study indicated that anxiety and depression levels increased with increasing demands and decreasing control and support scores ${ }^{10)}$.

Therefore, the aim of this study was to develop the Japanese translated version of DCSQ (J-DCSQ) and examine its reliability and validity.

\section{Subjects and Methods}

This study was approved by the Ethical Review Board of Fujita Health University School of Medicine.

\section{Subjects and Procedure}

Voluntary participants were enrolled from 19 nursery schools in Nagoya, Japan. Nursery schools are care facilities for children aged 0-6 years whose parents work during the day. The participants were required to fill in selfadministered questionnaires twice at an interval of 1 week. The 1st questionnaire included the J-DCSQ (1st J-DCSQ), Japanese version of the JCQ (J-JCQ) ${ }^{3,4)}$, and demographic variables. The 2 nd questionnaire contained only the JDCSQ (2nd J-DCSQ). Of the 356 participants who gave informed consent, $269(76 \%)$ responded to the questionnaires and sent back. After excluding $30(8 \%)$ women who did not respond to the J-DCSQ and/or J-JCQ, 26 (7\%) men, and one unknown respondent, we analysed the data from $212(60 \%)$ women. The reason was that, compared to women $(212(60 \%)$, fewer men $(26(7 \%))$ were available for analysis. The study's confidentiality was indicated in the documents given to the subjects. The characteristics of the subjects are shown in Table 1.

\section{Study Variables}

\section{J-DCSQ (see Appendix)}

The authors developed the J-DCSQ using the following process in order to ensure its content validity. One of the authors (Yuichiro Ono) translated the English- and Swedish-DCSQ into Japanese, which was followed by backtranslation into English by an independent professional translator. The authors sent these works to Dr. Theorell, the inventor of the DCSQ, for a review. Additional alterations were made in response to his comments.

The J-DCSQ consists of the following 3 subscales: PD (assessed with 5 items), DL (6 items), and SS (6 items). DL includes decision authority (DA: 2 items) and skill discretion (SD: 4 items). For PD and DL items, the respondents chose 1 of the following 4 frequency-based options: 'often', 'sometimes', 'occasionally', and 'never or hardly ever' (scored as 4, 3, 2, and 1, respectively). For each SS item, the following options are offered as possible responses: 'completely true', 'true to some extent', 'slightly untrue', and 'completely untrue' (scored as 4, 3, 2, and 1, respectively). Subscale scores equal the sum of the scores of the relevant items. Moreover, the scores are reversescored for the PD item number 4 'enough time' and DL item number 9 'repetitive work' when calculating the sub- 
Table 1. Characteristics of the subjects $(n=12)$

\begin{tabular}{|c|c|c|}
\hline Variable & $\mathrm{n}$ & $\%$ \\
\hline \multicolumn{3}{|l|}{ Age } \\
\hline $20-29$ & 72 & 34 \\
\hline $30-39$ & 36 & 17 \\
\hline $40-49$ & 54 & 25 \\
\hline $50-59$ & 46 & 22 \\
\hline $60-69$ & 4 & 2 \\
\hline \multicolumn{3}{|l|}{ Occupation } \\
\hline Nursery teacher & 175 & 83 \\
\hline Cook & 31 & 15 \\
\hline Nurse & 5 & 2 \\
\hline No response & 1 & 1 \\
\hline \multicolumn{3}{|c|}{ Working hours per week } \\
\hline $4-39$ & 92 & 43 \\
\hline $40-56$ & 94 & 44 \\
\hline No response & 26 & 12 \\
\hline \multicolumn{3}{|l|}{ Years of employment } \\
\hline $0-9$ & 111 & 52 \\
\hline $10-19$ & 29 & 14 \\
\hline $20-29$ & 44 & 21 \\
\hline $30-39$ & 19 & 9 \\
\hline No response & 8 & 4 \\
\hline \multicolumn{3}{|l|}{ Employment status } \\
\hline Permanent staff & 158 & 75 \\
\hline Temporary staff & 51 & 24 \\
\hline No response & 2 & 1 \\
\hline
\end{tabular}

scale scores. The reverse-score item for the DA of the JCQ was not adopted for the DCSQ. The non-adoption summarized the items and briefed the scale would contribute to reduce the necessary answering time. Greater subscale scores indicate higher levels of PD, DL, and SS.

\section{J-JCQ}

For the evaluation of the concurrent validity of the JDCSQ, the J-JCQ ${ }^{3,4)}$ was introduced as an established comparison for evaluating the psychosocial work environment based on the DCS model. Similar to the J-DCSQ, the J-JCQ consists of the following 3 subscales: PD (5 items), DL (9 items), and SS ( 8 items). Responses to all items are in the agree-disagree format: 'strongly agree', 'agree', 'disagree', and 'strongly disagree'. The scoring formulae, reliability, and validity were established ${ }^{3,4)}$. As in the JDCSQ, higher subscale scores indicate greater levels of $\mathrm{PD}$, DL, and SS.

\section{Demographic Variables}

The demographic variables included age and oc- cupational conditions. For occupational conditions, the subjects reported their occupations (nursery teacher, cook, or nurse), the average number of hours they worked per week, and the number of years they had been working for the nursery school (Table 1).

\section{Statistical methods for evaluating reliability and validity of the J-DCSQ \\ Reliability was evaluated with 1 -week test-retest reli-} ability and internal consistency. The authors calculated weighted kappa and intraclass correlation coefficients for the assessment of 1-week test-retest reliability using the data from the 1st and 2nd J-DCSQ. The weighted kappa method was adopted to measure the degree of agreement in each item score. Intraclass correlation was examined to determine the extent to which each subscale score agreed. Internal consistency was examined with Cronbach's alpha, using the data of the 1st J-DCSQ.

With regard to validity, we examined scaling, concurrent, and factorial validity. For evaluating the scaling validity, the ceiling and floor effects were examined for each subscale of the 1st J-DCSQ. The judgment condition was whether the values of mean added/subtracted standard deviations were within theoretically possible ranges, which were 5-20, 6-24, and 6-24 for PD, DL, and SS respectively. To evaluate concurrent validity, Spearman's correlation coefficients were calculated between the commensurate subscale scores of the 1st J-DCSQ and the J-JCQ. For the assessment of factorial validity, the exploratory factor analysis using the orthogonal rotation (principal axis factoring) method was used to obtain an uncorrelated and easily interpretable factorial structure ${ }^{7)}$. To evaluate the scree plot and the Eigenvalues, we obtained the number of factors ${ }^{15)}$. All J-DCSQ items were analysed simultaneously using factor analysis to confirm whether the questionnaire's subscale construct was the same as that of Swedish DCSQ. A factor loading as large as 0.35 or greater was considered acceptable ${ }^{15,16}$. Because Stevens recommended that investigators stop blindly using the rule of interpreting factors with a factor loading and consider the sample size, we calculated this cut-off point of a factor loading from the sample size ${ }^{16)}$.

In the present study, we could not calculate convergent and divergent validity, because we adopted only the DCSQ and the JCQ. Unanalysed divergent validity means that a portion of validity was not substantiated.

The subjects completed the anonymous questionnaire by answering individually and submitted their responses after putting them in an envelope and sealing it. The effect 
Table 2. Weighted kappa coefficients of the Japanese translated version of Demand-Control-Support Questionnaire

\begin{tabular}{lrlr}
\hline \multicolumn{1}{c}{ Subscale } & & \multicolumn{1}{c}{ Items } & $\begin{array}{c}\text { Weighted Kappa coefficients } \\
(95 \% \text { CI })\end{array}$ \\
\hline Psychological demands (PD) & 1 & work quickly & $0.72(0.55-0.90)$ \\
& 2 & work intensively & $0.65(0.41-0.89)$ \\
& 3 & work effort & $0.72(0.56-0.87)$ \\
& 4 & enough time & $0.66(0.51-0.81)$ \\
Decision latitude (DL) & 5 & conflicting demands & $0.67(0.49-0.86)$ \\
Skill discretion (SD) & & & \\
& 6 & learn new things & $0.60(0.26-0.85)$ \\
Social support (SS) & 7 & require skills \& ability & $0.64(0.39-0.80)$ \\
& 8 & require imagination \& ingenuity & $0.59(0.37-0.77)$ \\
& 9 & repetitive work & $0.61(0.41-0.81)$ \\
& 10 & how to do the work & $0.74(0.63-0.86)$ \\
& 11 & what work to do & $0.75(0.40-0.86)$ \\
& 12 & calm \& comfortable atmosphere & $0.60(0.30-0.80)$ \\
& 13 & relationships at work & $0.65(0.32-0.98)$ \\
& 14 & colleagues support & $0.58(0.24-0.92)$ \\
& 15 & helpful colleagues & $0.70(0.40-0.99)$ \\
& 16 & relationship with superiors & $0.65(0.34-0.97)$ \\
& 17 & good time with colleagues & $0.62(0.25-0.95)$ \\
\hline
\end{tabular}

of social desirability, however, was not investigated.

Acquiescence is vulnerable to the agree-disagree format and lowers the validity. To evaluate acquiescence, we examined whether the participants consistently agreed to all items of the J-JCQ. The same responding tendency was also evaluated for the J-DCSQ.

The analyses were conducted using SPSS 15.0 J (SPSS JAPAN Inc., Tokyo, Japan).

\section{Results}

Regarding the 1-week test-retest reliability of the JDCSQ, 15 items exhibited weighted kappa coefficients as large as 0.60 or greater (Table 2), and 2 items, a bit lower than 0.60 ( 0.58 and 0.59$)$. The intraclass correlation coefficients were 0.81 (95\% confidence interval (CI): 0.75-0.85), 0.82 (95\% CI: 0.77-0.86), and 0.80 (95\% CI: $0.75-0.84)$ for PD, DL, and SS respectively.

With regard to internal consistency, Cronbach's alphas (mean inter-item correlation) were 0.64 (0.26), 0.63 (0.22), and $0.87(0.50)$ for PD, DL, and SS respectively.

The score distribution of the 1st J-DCSQ in terms of scaling validity is shown in Fig. 1. The means added/subtracted SDs of all subscales were within theoretically possible score ranges. The distributions on extreme responses for PD and DL items were $46.3 \%$ in J-DCSQ ('often' or 'never or hardly') and 24.7\% in J-JCQ ('strongly agree' or 'strongly disagree').

None of the subjects consistently agreed to all items of either the J-JCQ or the J-DCSQ.

Regarding concurrent validity, Spearman's correlation coefficients were 0.74 (95\% CI: 0.67-0.80), 0.60 (95\% CI: 0.51-0.68), and 0.59 (95\% CI: 0.49-0.69) for PD, DL, and SS respectively.

With regard to factorial validity, the factor analysis was performed with principal axis factoring, Varimax rotation. Although the 4 factors had been extracted by Eigenvalue (1.088), the scree plot clearly indicated that 3 factors was acceptable. The 4 th factor was explaining only $6.40 \%$ of the variance. The complete set of SS items was united in Factor 1. Factor 2 consisted of a combination of PD and SD items. Factor 3 consisted of a complete set of DA items (Table 3).

The results remained unchanged even when the subjects were classified by age and occupations for analysis (data not shown).

\section{Discussion}

The aim of this study was to investigate the reliability and validity of the J-DCSQ, which had fewer items than the J-JCQ and used frequency format instead of the agree- 


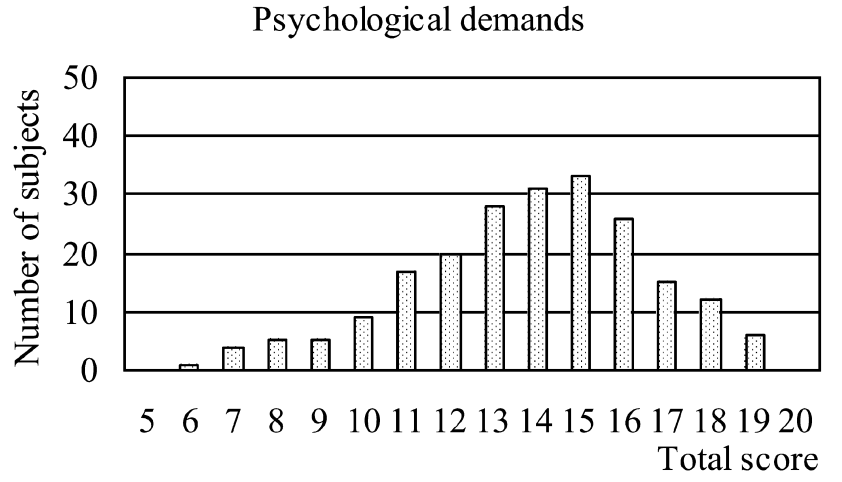

Mean (Standard deviation): 13.8 (2.7)

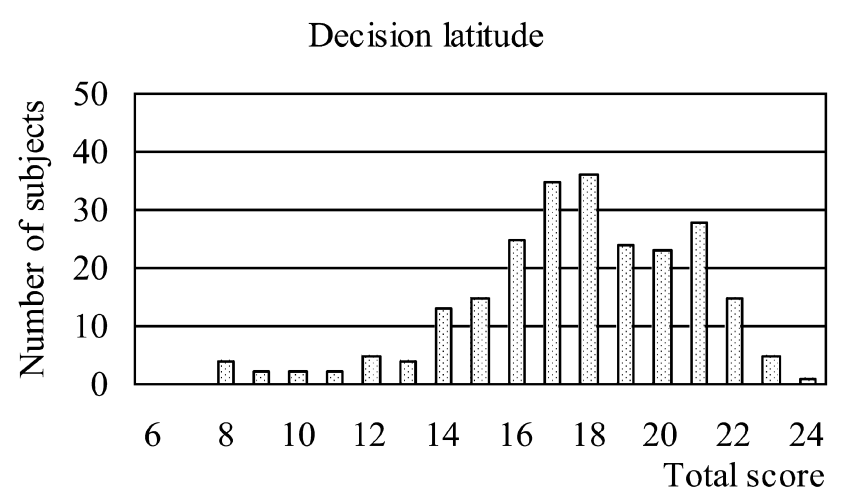

Mean (Standard deviation): 17.5 (3.1)

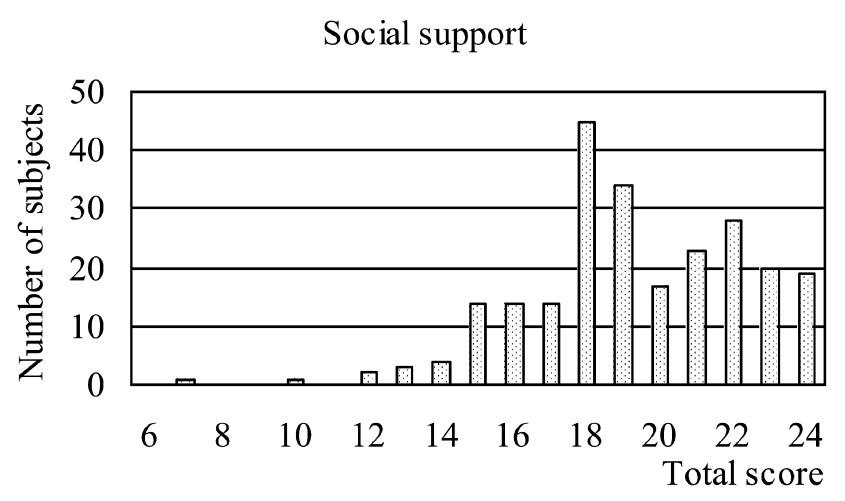

Mean (Standard deviation): 19.5 (2.9)

Fig. 1. Score distributions of the Japanese translated version of Demand-Control-Support Questionnaire subscales

disagree format.

The present findings suggested the reliability of the JDCSQ at a satisfactory level. Values of weighted kappa had moderate to adequate reliability as they were near or above 0.6. Intraclass correlation coefficients were high enough to approve the test-retest reliability. Internal consistency was at an adequate level, because the mean inter-item correlations were in the ideal range $(0.20-0.40)$ rather than at a moderate level of the Cronbach's alpha coefficients for PD and DL. PD and DL did not have high internal consistency like SS in this study. A similar tendency was observed in previous studies with regard to the DCSQ in other languages. Alpha coefficients ranged from $0.68-0.75$ for $\mathrm{PD}, 0.50-0.77$ for $\mathrm{DL}$, and $0.77-0.84$ for $\mathrm{SS}^{5,6,8,11,13)}$. Hence, the questionnaire's translation into Japanese itself did not contribute to the lower internal consistency of PD and DL. As we discuss later, factor analysis in this study did not indicate that PD and SD were distinct concepts from each other. This might contribute to the low internal consistency of PD and DL.

The present findings also suggested satisfactory scaling and concurrent validity of the J-DCSQ. The scaling validity was well established: no subscales exhibited ceiling/ floor effects. The J-DCSQ reflected the subjects' latent variable more adequately than did the J-JCQ, because of sufficient distribution on the extreme responses. The concurrent validity was approved: all the subscales indicated high Spearman's correlation coefficients. Since all the data were collected by means of self-reporting in the present study, common method bias could inflate the correlation. It is widely accepted that correlations between variables measured with the same method are inflated due to the action of common method variance (i.e., variance that is attributable to the measurement method itself, rather than to the constructs the measures represent) ${ }^{17,18)}$. Controversy remains over the extent to which the impact of common method variance should be considered. To avoid this question, we should have addressed convergent and divergent validity, for example. Convergent validity should be provided by assessing the Pearson correlation coefficient between the subscale of the relevant DCSQ and that of another scale (e.g. NIOSH-Generic job stress questionnaire (NIOSH-GJSQ) or Brief job stress questionnaire). Divergent validity could be confirmed by evaluating the Pearson correlation coefficient between the subscale of an unrelated DCSQ and that of another scale (e.g. physical demand and job insecurity of the JCQ (JCQ subscales which were not included in the J-JCQ)).

In contrast, factorial validity of the J-DCSQ was not demonstrated in this study as the inventor originally hypothesized.

One remarkable finding on the factorial validity was that PD and SD were not definitely separated. A similar finding was also observed in a study by Kawakami et al., in which the validity of the J-JCQ was examined ${ }^{3)}$. They 
Table 3. Exploratory factor analysis of the Japanese translated version of Demand-ControlSupport Questionnaire

\begin{tabular}{|c|c|c|c|c|c|}
\hline \multirow{2}{*}{ Subscale } & \multirow{2}{*}{\multicolumn{2}{|c|}{ Items }} & \multicolumn{3}{|c|}{ Factor } \\
\hline & & & 1 & 2 & 3 \\
\hline \multirow{5}{*}{ Psychological demands (PD) } & 1 & work quickly & & 0.49 & \\
\hline & 2 & work intensively & & 0.62 & \\
\hline & 3 & work effort & & 0.68 & \\
\hline & 4 & enough time & & 0.37 & \\
\hline & 5 & conflicting demands & & & \\
\hline \multicolumn{6}{|l|}{ Decision latitude (DL) } \\
\hline \multirow[t]{4}{*}{ Skill discretion (SD) } & 6 & learn new things & & 0.38 & \\
\hline & 7 & require skills \& ability & & 0.56 & \\
\hline & 8 & require imagination \& ingenuity & & 0.67 & \\
\hline & 9 & repetitive work & & & \\
\hline \multirow[t]{2}{*}{ Decision authority (DA) } & 10 & how to do the work & & & 0.85 \\
\hline & 11 & what work to do & & & 0.93 \\
\hline \multirow[t]{6}{*}{ Social support (SS) } & 12 & calm \& comfortable atmosphere & 0.67 & & \\
\hline & 13 & relationships at work & 0.73 & & \\
\hline & 14 & colleagues support & 0.76 & & \\
\hline & 15 & helpful colleagues & 0.72 & & \\
\hline & 16 & relationship with superiors & 0.64 & & \\
\hline & 17 & good time with colleagues & 0.73 & & \\
\hline Explained variance (\%) & & & 23.3 & 20.3 & 8.5 \\
\hline
\end{tabular}

hypothesized that, in some occupations, workers would require high-levelled skills and creativity to deal with job tasks involving qualitative and intellectual demands and that PD measured with the JCQ would contain not only quantitative but also qualitative and intellectual demands. Their hypothesis may be applied to our study findings. Most subjects in our study were nursery school teachers. Indeed, their work involves not only a series of fixed routines but also unexpected events in dealing with children. The latter would cause qualitative and intellectual demands that can be dealt using high-levelled skills and creativity. However, this hypothesis may be applicable to only Japanese employees in some occupations. In fact, previous studies proved the factorial validity of the Brazilian and Norwegian translated versions of the DCSQ, as originally hypothesized ${ }^{8,12)}$. Therefore, factorial validity has to be further examined among Japanese employees in occupations other than nursery school workers.

Another noteworthy finding of the factorial validity was low factor loadings of a PD item and a DL item concerning 'conflicting demands' and 'repetitive work' respectively. Investigations on the validity of the Brazilian and Swedish translated versions of the DCSQ found similar results as well ${ }^{8,12)}$. These findings could possibly be a result of the differences in item wording and response categories between the DCSQ and the JCQ ${ }^{12)}$. Karasek et al. reported different frequency distribution for these PD and DL items when the DCSQ and JCQ were simultaneously applied ${ }^{19)}$. Besides, some previous studies that examined the factorial validity of the JCQ also indicated low and inconsistent factor loadings on the PD and DL items concerning 'conflicting demands' and 'repetitive work' respectively ${ }^{20)}$. Thus, the low factor loadings of the PD and DL items found in our study may not be attributable to the DCSQ itself, but instead to the concept of PD and DL. According to Karasek et al., the definition of PD is comprehensive and includes mental work load, organizational constraints on task completion, and conflicting demands ${ }^{20}$. This dimensional diversity would provide the DCS model with a universal application to workers in a wide variety of occupations. At the same time, this feature may be an extra specification in a study like ours in which the subjects' occupations were limited. In the present study, the subjects were almost completely occupied with only 2 kinds of occupations: nursery teaching and cooking. For them, 'conflicting demands' might not be a work-related psychosocial stressor like PD.

Although our findings raised questions on the validity of the J-DCSQ, it is true that it did not perform a thorough investigation of validity. For instance, since our study 
was not a longitudinal observation, we were not able to address predictive validity of the J-DCSQ. Studies have substantially demonstrated predictive validity of the JCQ with regard to stress-related health disorders such as cardiovascular diseases, mental disorders, work-related musculoskeletal disorders, and insomnia ${ }^{1,2,20-23)}$. On the other hand, to the best of our knowledge, no researcher has so far examined the predictive validity of the DCSQ.

Furthermore, we discuss our study's limitations with regard to the subjects. First, because the study was based on voluntary participation, sampling biases might exist in our study. In addition, of the 356 participants who gave informed consent, almost one-quarter did not return the questionnaire, and 8 percent were excluded because they did not respond to the DCSQ and/or the JCQ. Moreover, the subjects included only female nursery school workers, which might restrict the generalisability of the present findings to male nursery teachers and men and women in other occupations. It is necessary to examine in further research whether our findings can be applied to other workers in other occupations. Second, although we used the abovementioned methods for minimising the effect of social desirability, it could affect validity. Further, even though the reverse-score item for the DA in the JCQ was not adopted for the DCSQ, none of the subjects consistently agreed to all items of either the J-JCQ or the J-DCSQ. Thus the influence of acquiescence was negligible. Moreover, further study is needed to assess social desirability, convergent validity, and divergent validity.

As we mentioned in the Introduction, the DCSQ has a possible advantage over the JCQ owing to smaller number of items and responses to frequency-based options. In conclusion, it exhibited sufficient reliability of the JDCSQ. Although Cronbach's alphas for PD and DL were relatively low, the mean inter-item correlation was at an ideal level. Values of weighted kappa were adequately reliable. Intraclass correlation coefficients were high enough to approve the test-retest reliability. Scaling validity and concurrent validity were also well established. The JDCSQ led the real improvement of response distribution on extreme responses. The factorial validity showed three uncorrelated factors: SS, DA, and 'PD and SD', instead of SS, PD, and DL (SD and DA). Further studies are needed to establish its validity. Our study presented the possibility that if evidence was expanded as to the validity of the DCSQ, it would become an alternative questionnaire to evaluate psychosocial work environment on the basis of the DCS model.

\section{Acknowledgements}

The authors acknowledge Professor Töres Theorell for permitting the use of the DSCQ and his assistance in developing the J-DCSQ.

\section{References}

1) Seward JP, Larsen RC (2007) Occupational Stress. In: Current occupational \& environmental medicine, LaDou L (Eds.), 579-94, McGraw-Hill, New York.

2) Karasek R, Theorell T (1990) The psychosocial work environment. Basic Books, New York.

3) Kawakami N, Kobayashi F, Araki S, Haratani T, Furui H (1995) Assessment of job stress dimensions based on the job demands-control model of employees of telecommunication and electric power companies in Japan: reliability and validity of the Japanese version of the Job Content Questionnaire. Int J Behav Med 2, 358-75. [Medline] [CrossRef]

4) Kawakami N, Fujigaki Y (1996) Reliability and validity of the Japanese version of job content questionnaire: replication and extension in computer company employees. Ind Health 34, 295-306. [Medline] [CrossRef]

5) Theorell T, Perski A, Åkerstedt T, Sigala F, Ahlberg-Hultén G, Svensson J, Eneroth P (1988) Changes in job strain in relation to changes in physiological state. Scand J Work Environ Health 14, 189-96. [Medline] [CrossRef]

6) Landsbergis P, Theorell T (2000) Measurement of psychosocial workplace exposure variables. Occup Med. State Art Rev 15, 163-88.

7) Aasa U, Brulin C, Ängquist KA, Barnekow-Bergkvist M (2005) Work-related psychosocial factors, worry about work conditions and health complaints among female and male ambulance personnel. Scand J Caring Sci 19, 251-8. [Medline] [CrossRef]

8) Sanne B, Torp S, Mykletun A, Dahl AA (2005) The Swedish Demand-Control-Support Questionnaire (DCSQ): factorial structure, item analyses, and internal consistency in a large population. Scand J Public Health 33, 166-74. [Medline] [CrossRef]

9) Sanne B, Mykletun A, Dahl AA, Moen BE, Tell GS (2005) Testing the job Demand-Control-Support model with anxiety and depression as outcomes: The Hordaland health Study. Occup Med (Lond) 55, 463-73. [Medline] [CrossRef]

10) Alderling M, Theorell T, de la Torre B, Lundberg I (2006) The demand control model and circadian saliva cortisol variations in a Swedish population based sample (The PART study). BMC Public Health 6, 288. [Medline] [CrossRef]

11) Demiral Y, Soysal A, Can Bilgin A, Kiliç B, Unal B, Uçku R, Theorell T (2006) The association of job strain with coronary heart disease and metabolic syndrome in 
municipal workers in Turkey. J Occup Health 48, 332-8. [Medline] [CrossRef]

12) Hökerberg $Y H$, Aguiar OB, Reichenheim M, Faerstein E, Valente JG, Fonseca Mde J, Passos SR (2010) Dimensional structure of the demand control support questionnaire: a Brazilian context. Int Arch Occup Environ Health 83, 407-16. [Medline] [CrossRef]

13) Aguiar OB, Fonseca Mde J, Valente JG (2010) Testretest reliability of the Swedish Demand-Control-Support Questionnaire among industrial restaurant workers in the state of Rio de Janeiro, Brazil. Rev Bras Epidemiol 13, $1-10$.

14) Schaeffer NC, Presser S (2003) The science of asking questions. Annu Rev Sociol 29, 65-88. [CrossRef]

15) Tabachnick BG, Fidell LS (2007) Using multivariate statistics. Fifth Edition. 607-75, Allyn \& Bacon, New York.

16) Stevens JP (2009) Applied multivariate statistics for the social science. Fifth edition. 325-94, Routledge, New York.

17) Podsakoff PM, MacKenzie SB, Lee JY, Podsakoff NP (2003) Common method biases in behavioral research: a critical review of the literature and recommended remedies. J Appl Psychol 88, 879-903. [Medline] [CrossRef]

18) Spector PE (2006) Method variance in organizational research: truth or urban legend? Organ Res Methods 9, 221-32. [CrossRef]
19) Karasek R, Choi B, Ostergren P, Ferraio M, Smet P (2007) Testing two methods to create comparable scales scores between the job content questionnaire (JCQ) and JCQ-like questionnaires in the European JACE study. Int J Behav Med 14, 189-201. [Medline] [CrossRef]

20) Karasek R, Brisson C, Kawakami N, Houtman I, Bongers P, Amick B (1998) The Job Content Questionnaire (JCQ): An instrument for internationally comparative assessments of psychosocial job characteristics. J Occup Health Psychol 3, 322-55. [Medline] [CrossRef]

21) Kivimäki M, Virtanen M, Elovainio M, Kouvonen A, Väänänen A, Vahtera J (2006) Work stress in the etiology of coronary heart disease - a meta-analysis. Scand J Work Environ Health 32, 431-42. [Medline] [CrossRef]

22) Stansfeld S, Candy B (2006) Psychosocial work environment and mental health - a meta-analytic review. Scand J Work Environ Health 32, 443-62. [Medline] [CrossRef]

23) Ota A, Masue T, Yasuda N, Tsutsumi A, Mino Y, Ohara H, Ono Y (2009) Psychosocial job characteristics and insomnia: A prospective cohort study using the DemandControl-Support (DCS) and Effort-Reward Imbalance (ERI) job stress models. Sleep Med 10, 1112-7. [Medline] [CrossRef] 


\section{APPENDIX}

The Swedish Demand-Control-Support Questionnaire

Psychological demands

1. Do you need to work very quickly? (work quickly)

2. Do you need to concentrate intensively while you work? (work intensively)

3. Does your work demand excessive effort? (work effort)

4. Do you have enough time to complete all your tasks? (enough time)

5. Are you often asked to perform tasks that contradict with each other? (conflicting demands)

Decision latitude

Skill discretion

6. Do you learn new things through your work? (learn new things)

7. Do you need advanced skills and specialized abilities to do your work? (require skills \& ability)

8. Are you expected to use imagination and ingenuity in your work? (require imagination \& ingenuity)

9. Do you have to repeat the same tasks over and over again? (repetitive work)

Decision authority

10. Do you exert any influence over decisions about how your work should be carried out? (how to do the work)

11. Do you exert any influence over decisions about the nature of your work? (what work to do)

Social support

12. There is a calm and comfortable atmosphere in my workplace. (calm \& comfortable atmosphere)

13. Everyone in my workplace gets on well with each other. (relationships at work)

14. My colleagues support me. (colleagues support)

15. The people around me show some understanding when I am not feeling at my best. (helpful colleagues)

16. I have a good relationship with my superiors. (relationship with superiors)

17. I have a good time working with my colleagues. (good time with colleagues)

The authors are responsible for the above-written English translations. 